\title{
EDITORIAL
}

\section{El estudio de la capacidad adquirida para el suicidio en tiempos de pandemia}

\section{The study of the acquired capacity for suicide in times of pandemic}

\author{
Yuly Suárez-Colorado (iD) 1 , Manuel K. Molina (iD) ${ }^{2}$
}

1. Universidad Cooperativa de Colombia. Santa Marta, Colombia. Correo: yuli.suarezcol@ucc.edu.co - https://orcid.org/0000-0001-7715-3556

2. Fundación Salud para el Trópico. Santa Marta, Colombia. Correo: manuelkmolina@gmail.com - https://orcid.org/0000-0002-7438-2166

El suicidio es un fenómeno complejo, resultados de múltiples interacciones de factores biológicos, sociales, culturales, psicológicos, entre otros ${ }^{1}$. Sin embargo, el espectro de comportamientos suicidas no es continuo en todos los casos, es decir, no toda ideación suicida desencadena un suicidio consumado ${ }^{2}$, e incluso no todo historial de intentos de suicidio terminará en muerte por suicidio ${ }^{3}$. En este sentido, el interés por conocer los factores que median la ideación / acción conducen a la capacidad suicida ${ }^{4}$. La teoría interpersonal propone a la capacidad adquirida para el suicidio, como factor que puede explicar porque un individuo procede a la acción ${ }^{5}$.

La teoría interpersonal para el suicidio es una de las primeras teorías de ideación-acción ${ }^{5}$, fundamentada en la carga percibida y baja pertenencia, al mismo tiempo que las personas están expuestas a eventos altamente adversos o dolorosos. La capacidad adquirida para el suicidio se configura a través de la exposición a eventos adversos de forma repetida y potencialmente letal ${ }^{5}$. El suicidio puede ser tan abrumador que se requiere elevada tolerancia al dolor e intrepidez a la muerte ${ }^{6}$. La teoría argumenta que sería necesaria la capacidad adquirida para ejecutar el acto suicida?.

Algunas fuentes para obtener la capacidad para el suicido corresponde a la exposición a combates ${ }^{8}$, autolesión no suicida ${ }^{9}$, abuso ${ }^{10,11}$ e intento suicida ${ }^{12}$.
En general, la exposición a eventos altamente adversos y dolorosos sostenidos en el tiempo. Algunos sugieren que una vez adquirida, la capacidad de suicidio es estable e irreversible ${ }^{13}$. Los estudios aún son limitados para comprender la capacidad adquirida para el suicidio.

Por otra parte, la carga percibida se refiere a la convicción de ser una carga para la familia, amigos o la sociedad en general y la idea de que otros estarán mejor sin uno mismo ${ }^{7}$, mientras, la pertenencia frustrada es definida como el sentido de no pertenecer a un grupo como una familia o un círculo de amigos. Los estudios estiman que la carga percibida y el sentido de no pertenencia se originan de la soledad y ausencia de reciprocidad o cuidados, así también los anteriores estados psicológicos se encuentran asociados a la ideación suicida ${ }^{13-15}$, a un mayor número intentos suicidas en pacientes ${ }^{16,17}$, soldados $^{18,19}$, y personal de la Fuerza Aérea ${ }^{20}$. En síntesis, el deseo de muerte, tolerancia al dolor, intrepidez a la muerte, carga percibida y una pertenencia frustrada representan factores que podrían explicar la ideación-acción.

Generalmente, el estudio del suicidio se enfoca en factores de riesgo o factores protectores para generar modelos predictivos, no obstante, la transición entre pensar y ejecutar el acto podría explicarse por la capacidad adquirida. En el contexto de la sindemia por COVID-19, la exposición continua a eventos dolorosos por las pérdidas familiares, 
económicas, sociales, afectivas pueden ser una fuente relevante para desarrollar alta tolerancia al dolor. Particularmente, el estrés crónico continuo o miedo por el riesgo al contagio o muerte, la restricción de interacciones sociales y las necesidades básicas insatisfechas pueden generar menor percepción de riesgo, en consecuencia, respuestas intrépidas hacia la muerte relacionadas con diversos comportamientos nocivos para la salud.

Es necesario reconocer que los determinantes sociales en salud, actualmente configuran un escenario de necesidades interpersonales en contexto de pandemia. Las desigualdades socialeseconómicas derivadas de la crisis global, multiplican el riesgo de valoración como carga para el entorno y experimentar no pertinencia social, enfoque de corte sociológico conocido por Durkheim como la necesidad de integración social.

Ahora bien, los datos epidemiológicos disponibles de 21 países al inicio de la pandemia mostraron que no se generaron incrementos en las tasas de suicidio, mientras en algunos casos la tendencia fue menor en este evento de salud pública ${ }^{21}$. Es importante considerar que la naturaleza de los comportamientos suicidas aún requiere de investigaciones que describan mejor las trayectorias, desde el conocimiento de un evento dinámico, complejo, multicausal. La sugerencia del suicidio como problema no automático y traducido directo a los factores de riesgo está presente en contexto de pandemia, por el contrario, la estimación de interacción, simultaneidad, y acumulación de factores de riesgo en determinado periodo de tiempo es próxima al curso de los comportamientos suicidas a largo plazo ${ }^{21}$. La naturaleza del curso de este evento en contexto de pandemia, no es completamente conocida.

Las limitaciones aún son significativas desde las políticas, investigación, e intervención, en medio de una emergencia de salud con repercusiones relevantes en la funcionalidad individual $y$ colectiva $^{22}$. Priorizar la prevención del suicidio en contexto de pandemia e incluso posterior a la pandemia es un reto global, así como el estudio e implementación de las medidas efectivas dado el estado actual de determinantes sociales en diversos contextos culturales ${ }^{1,22}$. Otra perspectiva, establece que son necesarias medidas eficientes en relación al costo-inacción, costo-acción, costo-eficacia, e incentivar la implementación de estrategias para la prevención ${ }^{23}$.

Finalmente, la posibilidad de configuración de capacidad adquirida a largo plazo en la población y especialmente en los países de bajos ingresos debe ser considerada, dada la acumulación de estresores que demandan de una colaboración de las ciencias de la salud y las ciencias sociales. El estudio de la capacidad adquirida para el suicidio adquiere pertinencia para la investigación-práctica, ante un evento prevenible ahora ${ }^{22,24}$.

\section{DECLARACIÓN SOBRE CONFLICTOS DE INTERÉS}

Ninguno que declarar.

\section{REFERENCIAS BIBLIOGRÁFICAS}

1. Campo-Arias A, Suárez-Colorado Y. ¿Es el suicidio un evento prevenible? Salud UIS. 2019; 51(3): 18799. Doi: https://doi.org/10.18273/revsal.v51n32019002

2. Klonsky E, Qiu T, Saffer B. Recent advances in differentiating suicide attempters from suicide ideators. Current Opinion in Psychiatry. 2017; 30(1): 15-20.

Doi: https://doi.org/10.1097/YCO.0000000000000294

3. Ribeiro J, Witte T, Van Orden K, Selby E, Gordon K, Bender $\mathrm{T}$, Joiner Jr, T. E. Fearlessness about death: The psychometric properties and construct validity of the revision to the Acquired Capability for Suicide Scale. Psychological Assessment. 2014; 26: 115-26. Doi: https://doi.org/10.1037/a0034858

4. Klonsky E., May A. Differentiating suicide attempters from suicide ideators: A critical frontier for suicidology research. Suicide and LifeThreatening Behavior. 2014; 44(1): 1-5. Doi: https://doi.org/10.1111/sltb.12068 
5. Van Orden K, Witte T, Cukrowicz K, Braithwaite S, Selby $E$, Joiner Jr, T. E. The interpersonal theory of suicide. Psychological review. 2010; 117(2): 575. Doi: https://doi.org/10.1037/a0018697

6. Gutierrez P, King C, Ghaziuddin N. Adolescent attitudes about death in relation to suicidality. Suicide and Life-Threatening Behavior. 1996; 26(1): 8-18. https://doi.org/10.1111/j.1943278X.1996.tb00252.x

7. Joiner TE. Why people die by suicide. Cambridge: Harvard University. Press; 2005. Disponible en: https://psycnet.apa.org/record/2011-29970-018

8. Butterworth S, Green B, Anestis M. The association between specific combat experiences and aspects of the Interpersonal Theory of Suicide. Comprehensive psychiatry. 2007; 78(7): 9-18. Doi: https://doi.org/10.1016/j.comppsych.2017.06.003

9. Matney J, Westers N, Horton S, King J, Eaddy M, Emslie G. J, et al. Frequency and methods of nonsuicidal self-injury in relation to acquired capability for suicide among adolescents. Archives of suicide research. 2018; 22(1): 91-105. Doi: https://doi.org/10.1080/13811118.2017.1283266

10. Allbaugh L, Florez I, Render Turmaud D, Quyyum N, Dunn S, Kim J, Kaslow N. J. Child abuse-Suicide resilience link in African American women: Interpersonal psychological mediators. Journal of aggression, maltreatment \& trauma. 2017; 26(10): 1055-071.

Doi: https://doi.org/10.1080/10926771.2017.1350773

11. Smith N, Monteith L, Rozek D, Meuret A. Childhood abuse, the interpersonal-psychological theory of suicide, and the mediating role of depression. Suicide and Life-Threatening Behavior. 2018; 48(5): 559-69. Doi: https://doi.org/10.1111/sltb.12380

12. Burke $\mathrm{T}$, Ammerman B, Knorr A, Alloy L, McCloskey M. Measuring acquired capability for suicide within an ideation-to-action framework. Psychology of violence. 2018; 8(2): 277. Disponible en: https://psycnet.apa.org/journals/vio/8/2/277/
13. Bryan C, Sinclair S, Heron E. Do military personnel "acquire" the capability for suicide from combat? A test of the interpersonal-psychological theory of suicide. Clinical Psychological Science. 2016; 4(3): 376-85. https://doi.org/10.1177/2167702615595000

14. Wachtel S, Teismann T. Die interpersonake theorie suizidalen verhaltens: Eine systematische übersichtsarbeit [La teoría interpersonal de la conducta suicida: una revisión sistemática]. Zeitschrift für Klinische Psychologie und Psychotherapie: Forschung und Praxis. 2013; 42(2): 96-106. Doi: https://doi.org/10.1026/1616$3443 / a 000193$

15. Ribeiro J. D. Joiner T. E. The interpersonalpsychological theory of suicidal behavior: Current status and future directions. Journal of clinical psychology. 2009; 65(12): 1291-299. Doi: https://doi.org/10.1002/jclp.20621

16. Van Orden K, Witte T, Gordon K, Bender T, Joiner Jr, TE. Deseo suicida y capacidad para el suicidio: pruebas de la teoría psicológica interpersonal de la conducta suicida entre adultos. Revista de consultoría y psicología clínica. 2008; 76(1): 72.

17. Anestis $M$, Joiner $T$. Examining the role of emotion in suicidality: Negative urgency as an amplifier of the relationship between components of the interpersonal-psychological theory of suicidal behavior and lifetime number of suicide attempts. Journal of affective disorders. 2011; 129(1-3): 26169. Doi: https://doi.org/10.1016/j.jad.2010.08.006

18. Bryan C, Cukrowicz K. Associations between types of combat violence and the acquired capability for suicide. Suicide and Life-Threatening Behavior. 2011; 41(2): 126-36. Doi: https://doi.org/10.1111/j.1943-278X.2011.00023.x

19. Bryan C, Morrow C, Anestis M, Joiner T. A preliminary test of the interpersonal-psychological theory of suicidal behavior in a military sample. Personality and Individual Differences. 2010; 48(3): 347-50.

Doi: 
20. Nademin E, Jobes D, Pflanz S, Jacoby A, Ghahramanlou-Holloway $M$, Campise $R$, et al. An investigation of interpersonal-psychological variables in Air Force suicides: A controlledcomparison study. Archives of Suicide Research. 2008; 12(4): 309-26. Disponible en: https://www.tandfonline.com/doi/abs/10.1080/13 811110802324847 ?casa_token $=0 \mathrm{Pq}$ (9HaDX8IAAAA A:iBWg-

58TBDHAzJterO1mNzarKHDE37df8v75qPHiHOupe8

A2LOW2tJ-_pOMkK2qjypzSJnRncSAcfOEvUw

21. Pirkis J, John A, Shin S, DelPozo-Banos M, Arya V., Analuisa-Aguilar P, Appleby L, et al. Suicide trends in the early months of the COVID-19 pandemic: An interrupted timeseries analysis of preliminary data from 21 countries. The Lancet Psychiatry. 2021; S221. Doi: https://doi.org/10.1016/S2215-0366(21)00091-2

22. Halabi-AIS, Fonseca-Pedrero E. Suicidal Behavior Prevention: The Time to Act is Now. Clínica y Salud. 2021; 32(2): 89-92. Disponible en: https://www.redalyc.org/journal/1806/180667951 007/html/

23. McDaid M. Making an economic case for investing in suicide prevention: Quo vadis? In R. C. O'Connor \& J. Pirkis (Eds.), The international handbook of suicide prevention (pp. 775-790). Wiley Blackwell; $2016 . \quad$ Disponible en: https://onlinelibrary.wiley.com/doi/abs/10.1002/9 781118903223.ch44

24. Knipe D, Hawton $K$, Siynor $M$, Niederkrotenthaler T. Researchers must contribute to responsible reporting of suicide. BMJ. 2021; 372:51. Doi: https://doi.org/10.1136/bmj.n351 